\title{
Ectopic ureter associated with uterine didelphys and obstructed hemivagina: preoperative diagnosis by MRI
}

\author{
Zhen J. Wang • Heike Daldrup-Link • \\ Fergus V. Coakley $•$ Benjamin M. Yeh
}

Received: 8 August 2009 /Revised: 18 September 2009 / Accepted: 19 October 2009 / Published online: 19 November 2009

(C) The Author(s) 2009. This article is published with open access at Springerlink.com

\begin{abstract}
Uterine didelphys with obstructed hemivagina and ipsilateral renal anomalies is a rare congenital malformation of the female urogenital tract. While the urinary anomalies almost always involve renal agenesis, we report a rare case of a 17-year-old girl with the malformation associated with ectopic ureteral insertion into the obstructed hemivagina, which was diagnosed preoperatively by MR imaging. To the best of our knowledge, preoperative MR imaging diagnosis of the ectopic ureter associated with this syndrome has not been previously reported. Accurate preoperative diagnosis of ectopic ureteral insertion associated with this syndrome is important for surgical planning.
\end{abstract}

Keywords Ureter · Ectopic · Mullerian duct · MRI ·

Adolescent

\section{Introduction}

The syndrome of uterine didelphys with obstructed hemivagina and ipsilateral renal anomaly (OHVIRA syndrome) is the result of lateral nonfusion of the Mullerian ducts with asymmetric obstruction. It is usually diagnosed in adolescence following menarche. The frequent presence of vaginal septum gives rise to unilateral hematocolpos, hematometra and sometimes hematosalpinx, resulting in abdominopelvic pain, especially during menstruation. The

Z. J. Wang $(\bowtie) \cdot$ H. Daldrup-Link $\cdot$ F. V. Coakley $\cdot$ B. M. Yeh Department of Radiology, University of California San Francisco, 505 Parnassus Ave.,

San Francisco, CA 94143-0628, USA

e-mail: jane.wang@radiology.ucsf.edu pain may or may not continue beyond menses depending on the degree of the hemivaginal obstruction. While the syndrome is almost always associated with renal agenesis, we present a girl with uterine didelphys with associated ectopic ureteral insertion into the obstructed hemivagina, which was diagnosed preoperatively by MR imaging.

\section{Case report}

A 17-year-old girl was referred for pelvic MRI because of persistent vaginal discharge. Her previous workup included sonography, which revealed a solitary right kidney as well as probable uterine didelphys. She began menstruating at the age of 13 and had complaints of dysmenorrhea, which has worsened over the years. In between her menses, she reported persistent clear vaginal discharge.

MR imaging through the abdomen and pelvis confirmed uterine didelphys with vaginal duplication, and obstructed left hemivagina distended with fluid (Fig. 1). In addition, it showed a dilated blind-ending left ureter with ectopic insertion into the obstructed left upper vagina (Fig. 1).

The girl subsequently underwent excision of the vaginal septum by a gynecologic surgeon to alleviate the left hemivagina obstruction. At the same time, she had vaginoscopy, which confirmed the ectopic insertion of the left ureter into the left hemivagina. No left renal remnant was visualized at surgery, and the girl underwent laparoscopic left ureterectomy performed by a urologic surgeon immediately following the vaginoplasty. The girl had an uncomplicated postoperative recovery with resolution of her previous symptoms. 

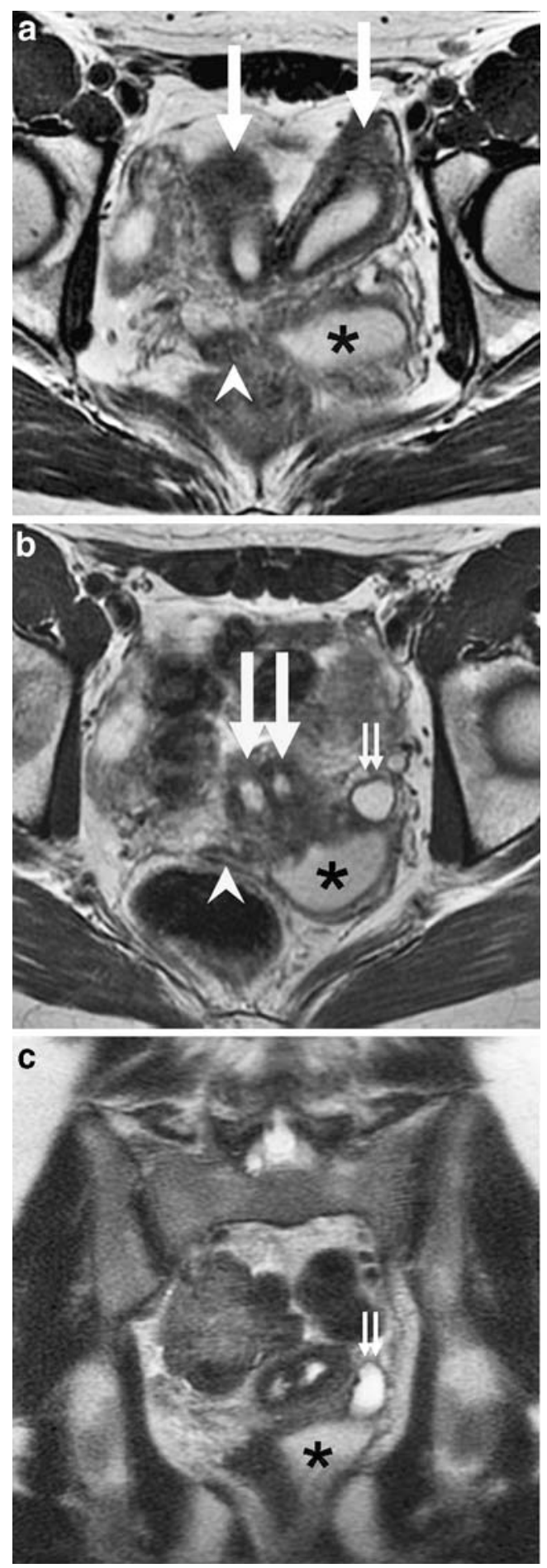

\section{Discussion}

Because of the close embryological association of the Wolffian and Mullerian anlage, renal anomalies, predominantly renal agenesis, are almost always seen in patients with uterine didelphys with obstructed hemivagina [1]. In a recent review of 23 cases with this constellation of findings, 20 patients had ipsilateral renal agenesis, 2 patients had
Fig. 1 Uterine didelphys, obstructed hemivagina, and ectopic ureter on MR imaging in a 17-year-old girl. a Axial T2-W image demonstrates two widely separate uterine horns (large arrows), an obstructed left hemivagina distended with fluid (asterisk), and a nondilated right hemivagina (arrowhead). b Axial T2-weighted image demonstrates two cervices (large arrows), an obstructed left hemivagina distended with fluid (asterisk), a nondilated right hemivagina (arrowhead), and a dilated left ureter (small arrows). On T1-W images, the fluid in the obstructed left hemivagina and the dilated left ureter was hypointense (not shown). c Coronal T2-W image demonstrates the dilated left ureter (small arrows) inserting ectopically into the obstructed left hemivagina (asterisk). There is absence of visible left renal tissue (not shown)

either dysplastic or polycystic kidney, and 1 patient had duplicated ureter [1]. Ectopic ureter associated with this syndrome is very rare and has only been reported in a few cases, with the ureter either inserting into the obstructed vaginal cavity or a Gartner's duct cyst [2-4]. The embryology of the ectopic ureter is unclear. Capito et al. [5] hypothesized that when an anomaly of the distal Wolffian duct is present, a unilateral anomalous incorporation of this duct within the bladder occurs, resulting in the anomalous incorporation of the attached ureteric bud within the bladder with subsequent ectopic (vaginal) ureteral insertion. This would lead to renal dysgenesis as the formation of the definitive kidney requires cranial growth of the ureteral bud [5].

Because of the rare occurrence of ectopic ureter associated with uterine didelphys and obstructed hemivagina, the diagnosis of ectopic ureteral insertion can be easily overlooked. In the two reports $[2,3]$ describing the association, the diagnosis of the ectopic ureteral insertion into the obstructed hemivagina was not made until the patients presented with persistent urine leakage through the vagina following vaginoplasty. In those cases, the patients had to be brought back to the operating room for the second time for excision of the ectopic ureter. It is, therefore, important to preoperatively make the diagnosis of the associated ectopic ureter as it may affect surgical planning. Normally, patients would undergo vaginoplasty with resection of the septum performed by a gynecologic surgeon to relieve the obstruction and pain. However, if an associated ectopic ureter is suspected, a urologist should then be present at the time of the vaginoplasty to search for the ectopic ureteral orifice and perform ureterectomy if appropriate. In our case, ureterectomy was performed, but a remnant of the left kidney was not visualized at surgery. We speculate that although the left renal remnant was not found, it was likely present given the symptoms of persistent vaginal discharge prior to surgery in conjunction with the ectopic ureter confirmed at surgery. Our patient likely had left renal dysgenesis with remnant that still had residual function and produced a small amount of urine, rather than true renal agenesis. The renal remnant has been 
reported to be ectopic in location [2], which may further increase the difficulty in locating it on imaging or at surgery.

Sheih et al. [4] reported a case of vaginal ectopic ureter inserted into an imperforate hemivagina that was detected by US. Sonography is frequently the first imaging modality in assessing genitourinary pathologies; it is readily available, inexpensive, and can provide excellent visualization of dilated ureters. Unfortunately, in our case, the ectopic ureter was missed on the sonogram. Preoperative MR imaging diagnosis of an ectopic ureter associated with the syndrome of uterine didelphys with obstructed hemivagina is unique. MR imaging is an excellent modality for evaluating the frequently complex genitourinary anomalies, given its mutiplanar capability, superior tissue characterization, and the lack of ionizing radiation. Suspected Mullerian duct anomalies are already frequently evaluated by MR imaging because of its superior ability in delineating uterine contour, shape of uterine cavity, and tissue characterization of the septa [6]. Several studies have also reported greater sensitivity of MR imaging compared to sonography or intravenous urography in diagnosing dysplastic kidneys with ectopic ureters [7]. MR imaging can provide detailed, multi-planar images of dysplastic renal units, such as ectopic ureteral insertion, that are both diagnostic and predictive of intraoperative findings in cases where other modalities have failed [8]. In our case, MR imaging allowed preoperative diagnosis of the associated ectopic ureteral insertion into the obstructed hemivagina; this facilitated operative planning such that the resection of the ectopic ureter could be performed at the same time of the vaginoplasty. Given the complexity of the Mullerian duct malformation, MR imaging may be useful preoperatively to assess the associated genitourinary anomalies. However, it would require larger future studies to evaluate whether preoperative MR imaging should be obtained in all such cases or whether it should only be used as a problem-solving tool when the diagnosis by sonography is equivocal.

In conclusion, it is important for radiologists to be aware of the rare association of an ectopic ureter with the syndrome of uterine didelphys with obstructed hemivagina. MR imaging may be useful in the preoperative diagnosis of the ectopic ureter, and may facilitate surgical planning.

Open Access This article is distributed under the terms of the Creative Commons Attribution Noncommercial License which permits any noncommercial use, distribution, and reproduction in any medium, provided the original author(s) and source are credited.

\section{References}

1. Smith NA, Laufer MR (2007) Obstructed hemivagina and ipsilateral renal anomaly (OHVIRA) syndrome: management and follow-up. Fertil Steril 87:918-922

2. Duong DT, Shortliffe LM (2008) A case of ectopic dysplastic kidney and ectopic ureter diagnosed by MRI. Nat Clin Pract Urol 5:632-636

3. Shibata T, Nonomura K, Kakizaki H et al (1995) A case of unique communication between blind-ending ectopic ureter and ipsilateral hemi-hematocolpometra in uterus didelphys. J Urol 153:1208-1210

4. Sheih CP, Chiang CD, Liao YJ et al (1997) Ultrasonic detection of a single vaginal ectopic ureter inserted into an imperforate hemivagina. J Clin Ultrasound 25:133-135

5. Capito C, Echaieb A, Lortat-Jacob S et al (2008) Pitfalls in the diagnosis and management of obstructive uterovaginal duplication: a series of 32 cases. Pediatrics 122:e891-897

6. Carrington BM, Hricak H, Nuruddin RN et al (1990) Mullerian duct anomalies: MR imaging evaluation. Radiology 176:715720

7. Borthne A, Pierre-Jerome C, Nordshus T et al (2000) MR urography in children: current status and future development. Eur Radiol 10:503-511

8. McMann LP, Kirsch AJ, Scherz HC et al (2006) Magnetic resonance urography in the evaluation of prenatally diagnosed hydronephrosis and renal dysgenesis. J Urol 176:1786-1792 